\title{
Mesure de débit en écoulement turbulent pulsé à l'aide d'un diaphragme
}

\section{Measurement of pulsating turbulent flow rate with an orifice plate}

\author{
Hubert Mainardi \\ Docteur Ingénieur \\ Laboratoire d'Energétique et de Mécanique Appliquée \\ B.P. 6057 - 45017 Orléans
}

\section{Introduction}

Lors de la détermination du débit masse en écoulement instationnaire il est préconisé, pour des taux de modulation de débit supérieurs à $10 \%$, de placer des capacités tampon en amont et en aval de l'appareil de mesure. Ces volumes amortissent les surpressions inhérentes aux modes de résonance des ondes de pression.

L'étude que nous présentons a pour but d'analyser l'influence de ces caractéristiques acoustiques sur l'évaluation du débit masse mesuré à l'aide d'un diaphragme noyé dans une canalisation cylindrique lorsque ces volumes sont supprimés.

\section{Dispositif expérimental}

L'étude expérimentale a été effectuée sur une installation de type industriel avec entrée à bord vif, puis avec une entrée profilée (fig. 1).

L'air est aspiré directement dans l'atmosphère par une soufflerie du type Eiffel à col sonique régulé. La modulation de débit est générée à partir d'une obturation partielle du col sonique par un profil entraîné mécaniquement. Ce dispositif permet d'obtenir :

- des fréquences pulsatoires comprises entre 0 et $60 \mathrm{~Hz}$.

- des amplitudes de modulation de débit comprises entre 0 et $50 \%$.

Le débit masse mesuré à partir de la perte de charge singulière du diaphragme

$$
Q_{m}=m C_{D_{n}} A \epsilon \sqrt{2 \rho \Delta p_{1,2}}
$$

est comparé à celui de la tuyère sonique

$$
Q_{m}=A_{c} K_{3} P_{i}^{(\gamma-1) / 2 \gamma}
$$

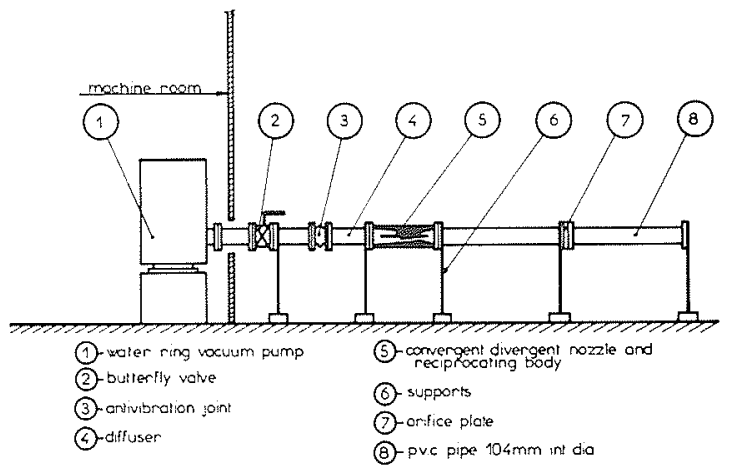

Figure 1

Ecoulement turbulent pulsé dans le cas d'une entrée à bord vif (fig. 2c)

Un modèle monodimensionnel résoud :

- l'équation d'Euler

$$
\frac{\partial u}{\partial t}+u \frac{\partial u}{\partial x}+\frac{1}{\rho} \cdot \frac{\partial p}{\partial x}+f u^{2}=0
$$

- l'équation de continuité

$$
\frac{\partial \rho}{\partial t}+\rho \cdot \frac{\partial u}{\partial x}+u \frac{\partial \rho}{\partial x}=0
$$

- l'équation de Laplace remplaçant l'équation d'énergie et tenant compte de l'hypothèse faite sur le comportement thermodynamique de l'écoulement: faible irréversibilité. 

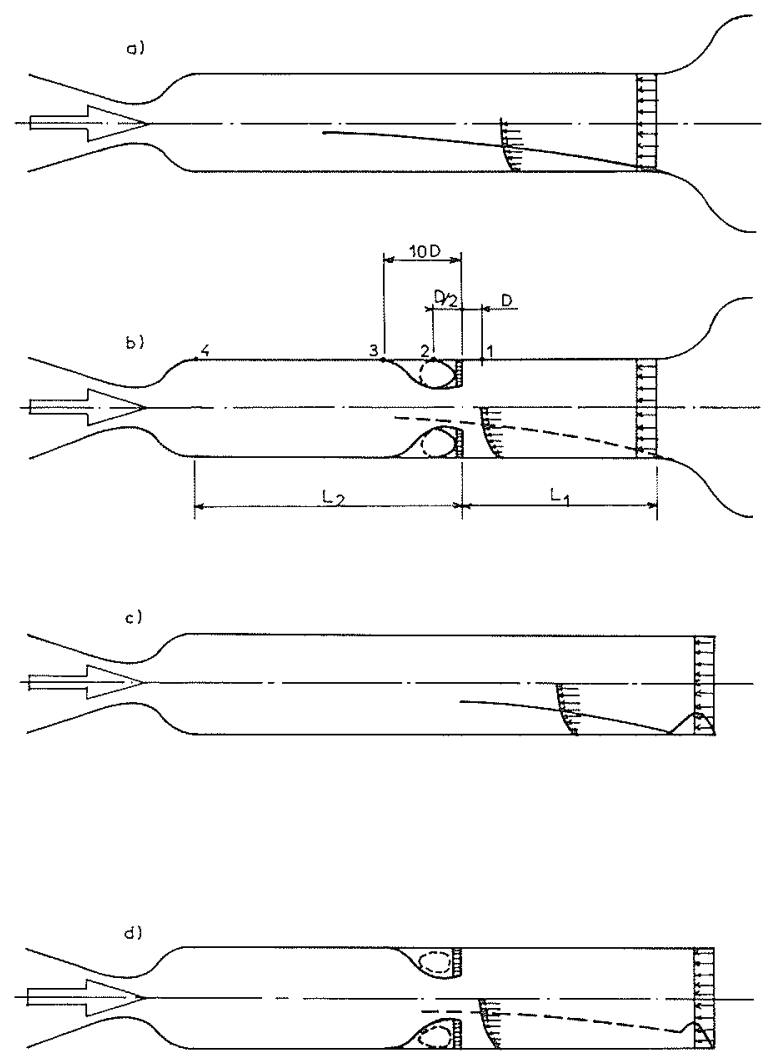

Figure 2

En résolvant ces équations par rapport à une vitesse de débit modulée a l'entrée de la canalisation [1]

$$
\begin{gathered}
\left|\widetilde{V}_{0}\right|=\psi\left|\bar{U}_{q}\right| \\
\psi=\frac{Q_{\max }-Q_{\min }}{Q_{\max }+Q_{\min }}
\end{gathered}
$$

la section sonique théorique correspondante est calculée $\left|\widetilde{A}_{c}\right|_{t h}$. La modulation de section sonique réelle étant mesurée, nous obtenons la modulation réelle de vitesse à l'entrée de la canalisation

$$
\left|\bar{V}_{r}\right|=\left|\widetilde{V}_{0}\right| \cdot \frac{\left|\widetilde{A}_{c}\right|_{r}}{\left|A_{c}\right|_{\text {th }}}
$$

Connaissant les grandeurs de l'écoulement à l'entrée de la canalisation le modèle calcule les fluctuations de pression de l'écoulement en toute abscisse $x$ du conduit.

\section{Résultats obtenus}

La superposition des courbes théoriques et expérimentales des fluctuations de pression en fonction des fréquences pulsatoires (fig. 3) est obtenue en donnant au coefficient $f$ de l'équation (1) différentes valeurs dépendantes du taux de modulation de débit (fig. 4). Ce coefficient $f$ englobe la perte de charge singulière d'entrée et la perte de charge régulière $\left(\xi_{c} / D\right)^{2}$. Cette dernière a été prise égale à 0,16 .

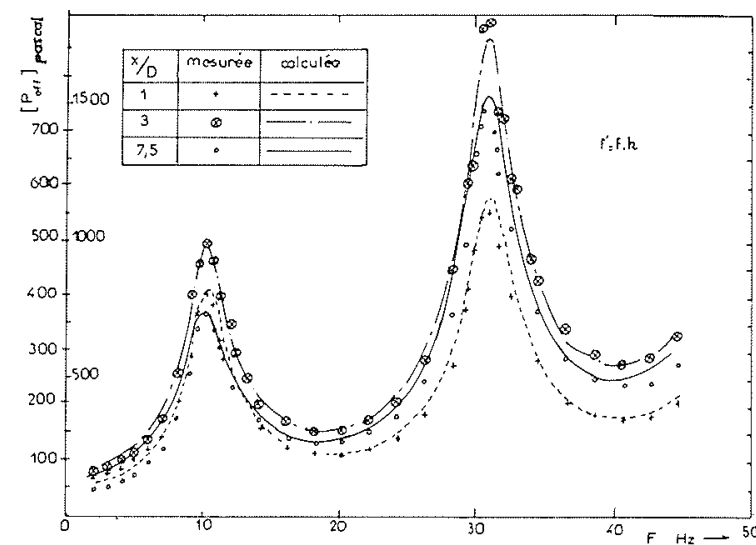

Figure 3

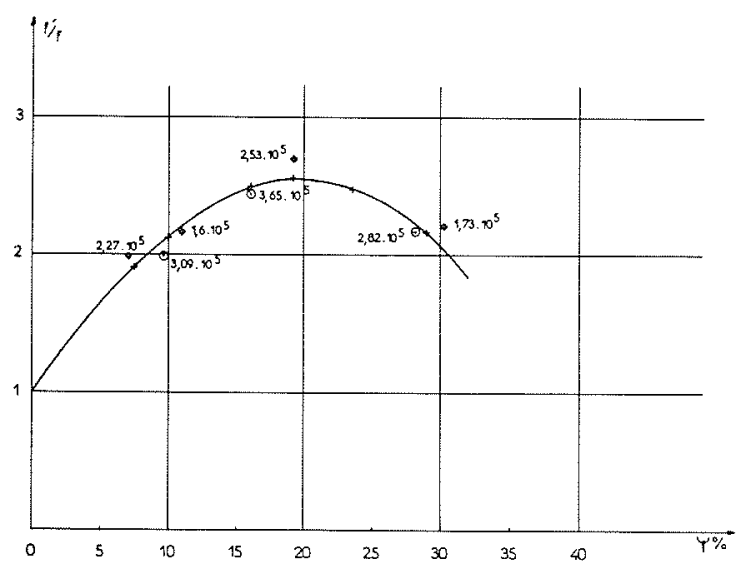

Figure 4

\section{Perte de charge singulière du diaphragme (fig. $2 \mathrm{~d}$ )}

Lorsque le diaphragme est placé dans la canalisation à une distance de l'entrée égale ou supérieure à 50 diamètres de conduit, c'est-à-dire dans le domaine où les profils de vitesse longitudinale sont répétitifs, le modèle monodimensionnel résoud, pour les longueurs de canalisation en amont et en aval du diaphragme, les équations 1 et 2 . Entre les plans (1) et (3) situés respectivement à un diamètre en amont du diaphragme et à 10 diamètres en aval de ce dernier le diaphragme est caractérisé par la loi suivante :

$$
\Delta p_{1,3}=\xi_{d} \cdot \rho_{1} \cdot \frac{u^{2}}{2}+L_{e} \cdot \rho_{1} \cdot \frac{\partial u}{\partial t}
$$

La distance $L_{e}$, égale à $10 \mathrm{D}$, en écoulement stationnaire, correspond au point de recollement du jet à la paroi de la canalisation.

\section{Résultats obtenus}

En considérant la perte de charge singulière du diaphragme $\left(\xi_{d}\right)$ constante, la superposition des courbes théoriques et expérimentales des fluctuations de pression dans le plan (1) en fonction des fréquences pulsatoires, (fig. 5), s'obtient en donnant au coefficient $f$ 


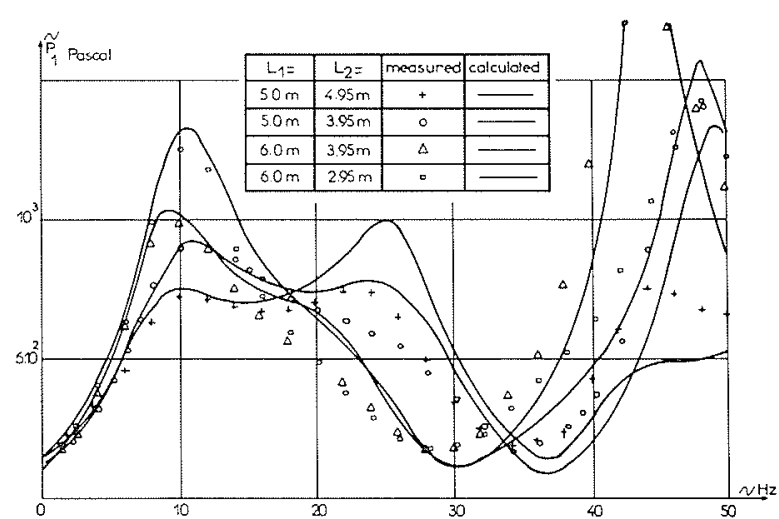

Figure 5

une valeur correspondante à celle de la perte de charge régulière majorée de $33 \%$. Lorsque le taux de modulation de débit est inférieur à $10 \%$, la longueur $L_{e}$ est identique à celle de l'écoulement stationnaire. Pour un taux de modulation de débit égal à $15 \%$ cette longueur est égale à $6 D$.

\section{Coefficient de débit}

Les fluctuations de débit dans le plan (1) déterminées, le coefficient de débit $\left(K_{0}=m C_{D n}\right)$ est calculé à partir de l'expression

$$
\Delta p_{1,2}=K_{0} \cdot \rho_{1} \cdot \frac{Q}{2 A^{2}}+\frac{\rho_{1}}{\ell} \cdot \frac{\partial}{\partial t}(k \cdot Q)
$$

\section{Résultats obtenus}

La superposition des courbes théoriques et expérimentales des fluctuations de pression dans le plan (2), situé à un demi diamètre de conduit en aval du diaphragme, en fonction des fréquences pulsatoires, (fig. 6), est obtenue :

- dans le cas d'un taux de modulation de débit égal à $6 \%$ en donnant au coefficient $K_{0}$ une valeur identique à celle qu'il a en écoulement stationnaire, - lorsque le taux de modulation de débit est égal à $15 \%$, le coefficient $K_{0}$ a une valeur dépendante du mode acoustique de l'installation. Cette modification correspond à la surestimation du débit moyen. Elle est de l'ordre de $1,5 \%$ lorsque $\psi$ est égal à $6 \%$, de l'ordre de $4,5 \%$ lorsque $\psi$ est égal à $15 \%$.

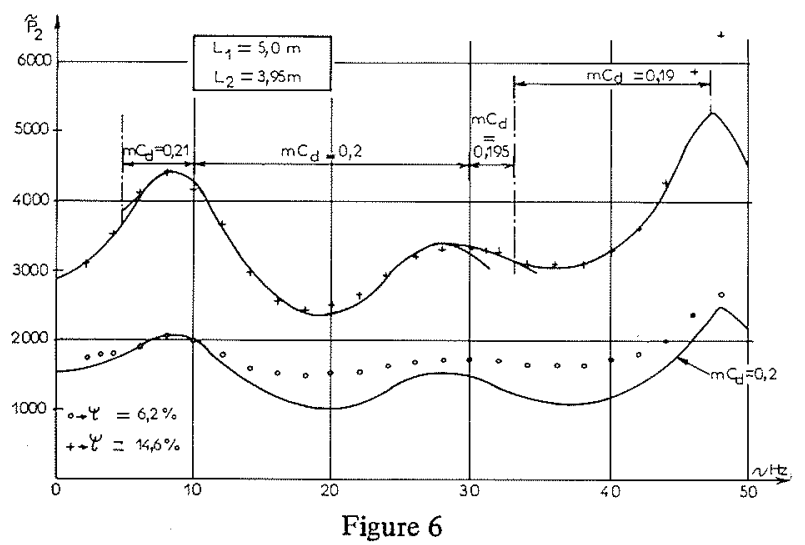

\section{Conclusion}

Cette modification du coefficient de débit semble ètre due aux ondes de pression au voisinage du diaphragme et aux effets locaux directs (respiration du bulbe en aval du diaphragme).

L'étude théorique de ce phénomène ne peut être effectuée qu'à partir d'un modèle bidimensionnel de l'écoulement rendant compte des grandeurs amont (vitesses, pression et contrainte tangentielle). Ces grandeurs connues, la modélisation du jet confiné permettrait d'analyser l'influence du taux de modulation de débit et des modes acoustiques sur le bulbe aval.

Le coefficient de frottement $f$ de cette étude englobant la perte de charge singulière de l'entrée à bord vif, il est nécessaire d'effectuer une étude similaire avec une entrée profilée. Ce type d'entrée permet d'obtenir un développement continu de la couche limite turbulente et la modélisation de l'écoulement correspondant.

Ecoulement turbulent pulsé. Perte de charge régulière

La modélisation du développement de la couche limite permet de déterminer le coefficient de perte de charge régulière utilisé dans l'expression du coefficient $f$ de l'équation (1). Cette modélisation est effectuée dans le cas de l'écoulement stationnaire. Les valeurs théoriques et expérimentales sont comparées.

Les modulations de pression calculées en écoulement turbulent pulsé sont comparées aux fluctuations de pression expérimentales.

\section{Ecoulement turbulent stationnaire}

Basé sur le modèle de Nelson et Pletcher [3] qui résoud:

- l'équation de Navier Stokes

$$
\bar{\rho}\left(\bar{u} \cdot \frac{\partial \bar{u}}{\partial x}+\bar{v} \frac{\partial \bar{u}}{\partial r}\right)=-\frac{\partial \bar{p}}{\partial x}+\frac{1}{r} \cdot \frac{\partial}{\partial r}\left(r \mu_{e f f} \frac{\partial \bar{u}}{\partial r}\right)
$$

où $\mu_{\text {eff }}$ dépend de la viscosité cinématique du fluide et de la longueur de mélange $\left(\ell_{1}\right)$

$$
\mu_{e f f}=\mu+\bar{\rho} \cdot \ell_{1}\left|\frac{\partial \bar{u}}{\partial r}\right|
$$

Lorsque $y \leqslant 0,089 \delta$ ( $\delta$ : épaisseur de couche limite) nous utilisons la longueur de mélange de Van Driest en écoulement stationnaire etabli (domaine des profils répétitifs)

$$
\ell_{1}=0,4\left(1-e^{-y^{+} / 26}+e^{-60 y^{+} / k_{1}^{+}}\right)
$$

Pour $y>0,089 \delta$ nous avons $\ell_{1}=0,089 \delta$ - l'équation de continuité

$$
\frac{\partial}{\partial x}(\bar{\rho} \cdot \bar{u})+\frac{1}{r} \cdot \frac{\partial}{\partial r}(\bar{\rho} \cdot \bar{v} \cdot r)=0
$$


- l'expression du débit masse

$$
m=\int_{A} \bar{\rho} \cdot \bar{u} \cdot d A=\text { const }
$$

Le gradient de pression longitudinal est déterminé par une boucle de calcul sur la détermination du débit masse à $10^{-5}$ près.

\section{Résultats obtenus}

Les grandeurs expérimentales (vitesse longitudinale et pression statique de paroi) sont comparées à celles calculées. Sur la figure 7 sont reportées les vitesses longitudinales en fonction de l'ordonnée $y$ comptée à partir de la paroi pour différentes abscisses $x$ égales à $2 D, 4 D, 6,25 D$ et $15 D$. Sur la figure 8 est reportée la pression statique $p$ en fonction de l'abscisse réduite $(x / D)$. Sur cette figure sont reportés le gradient de pression longitudinal et le coefficient de frottement $C_{f}$ calculés. Le coefficient de perte de charge régulière moyen, dans le domaine où le gradient de pression longitudinal évolue $(0 \leqslant x \leqslant 25 D)$, est déterminé par intégration graphique du coefficient de frottement dans ce domaine. Le coefficient $f$ correspondant est égal à 0,125 .

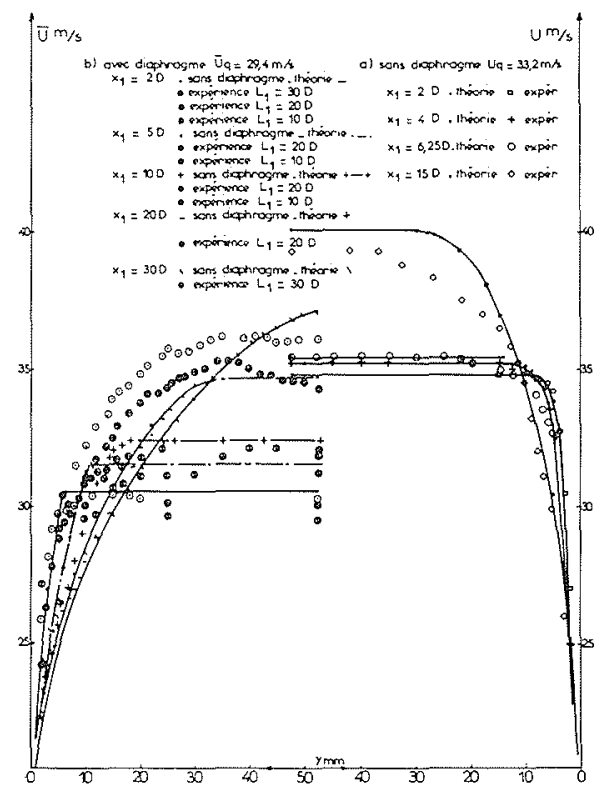

Figure 7

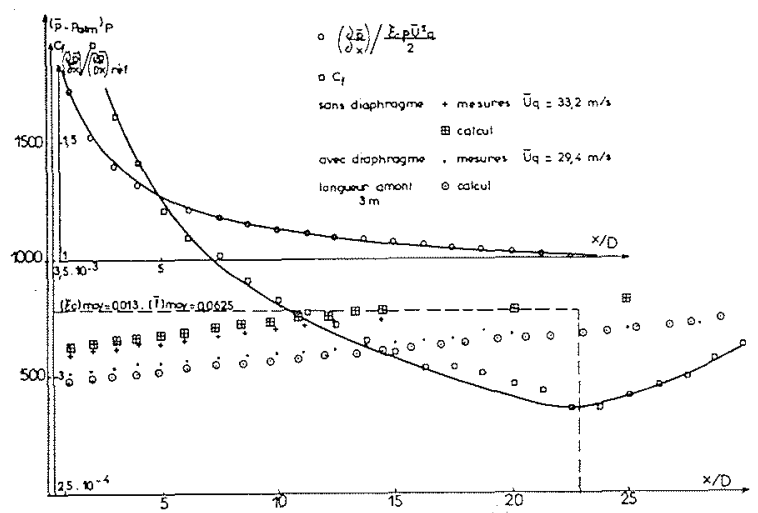

Figure 8
Sur ces figures ont été reportées les grandeurs expérimentales obtenues lorsque le diaphragme est placé dans la canalisation. Nous pouvons noter que la présence du diaphragme modifie :

- le développement de la couche limite, et son in-

fluence se ressent sur cinq diamètres en amont du diaphragme,

- l'évolution de la perte de charge régulière qui est majorée par la présence du diaphragme sur deux diamètres en amont de celui-ci.

Perte de charge régulière en écoulement turbulent pulsé (fig. $2 \mathrm{a}$ )

La perte de charge de l'écoulement moyen pulsé de vitesse égale à la vitesse moyenne est identique à celle de l'écoulement stationnaire ayant même vitesse moyenne.

La superposition des courbes expérimentales et théoriques des fluctuations de pression en fonction de l'abscisse réduite, pour les deux premiers modes de résonance, est obtenue en donnant au coefficient $f$ les valeurs indiquées sur la figure 9 . Ces valeurs dépendent du mode acoustique et du gradient de pression longitudinal. Elles sont indépendantes du taux de modulation de débit. Au premier mode le coefficient $f$ correspond à la perte de charge régulière $\left(\xi_{c} / D\right)$ en écoulement stationnaire dans la domaine établi quel que soit le gradient de pression; pour le $2^{\text {eme }}$ et $3^{\text {eme }}$ mode il a même valeur dans le domaine où le gradient de pression longitudinal est constant $(x>25 D)$. Lorsque ce gradient évolue $(0 \leqslant x \leqslant 25 D)$ le coefficient $f$ est majoré de $60 \%$ pour le $2^{\text {ème }}$ mode et de $50 \%$ pour le $3^{\text {eme }}$ mode.

La dépendance du coefficient $f$ au mode de résonance peut être attribuée :

- soit à la pression acoustique induite. L'étude expérimentale indique que les profils de vitesse longitudinale moyenne sont faiblement modifiés aux modes de résonance, ce qui entraînerait un changement d'épaisseur de couche limite. L'altération expérimentale est de l'ordre de $3 \%$. Celle-ci aurait pour conséquence de modifier l'équilibre des mécanismes turbulents bien que le nombre de Strouhal turbulent correspondant aux fréquences d'oscillations forcées

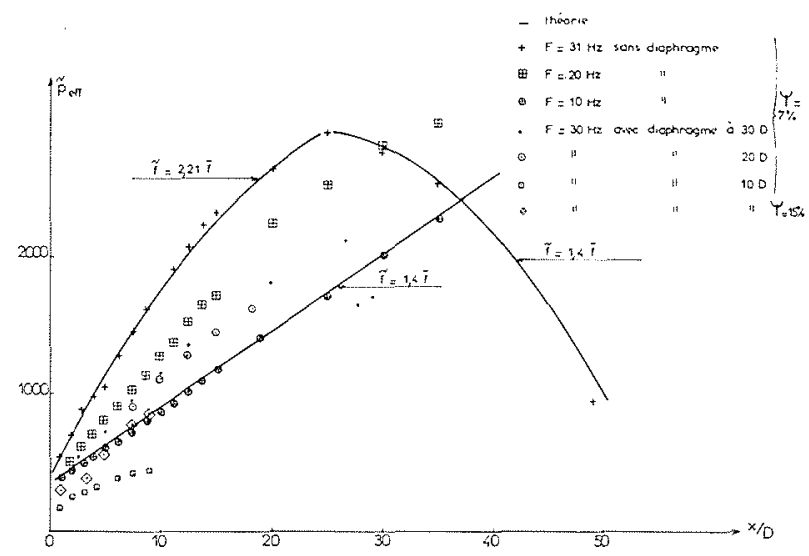

Figure 9 


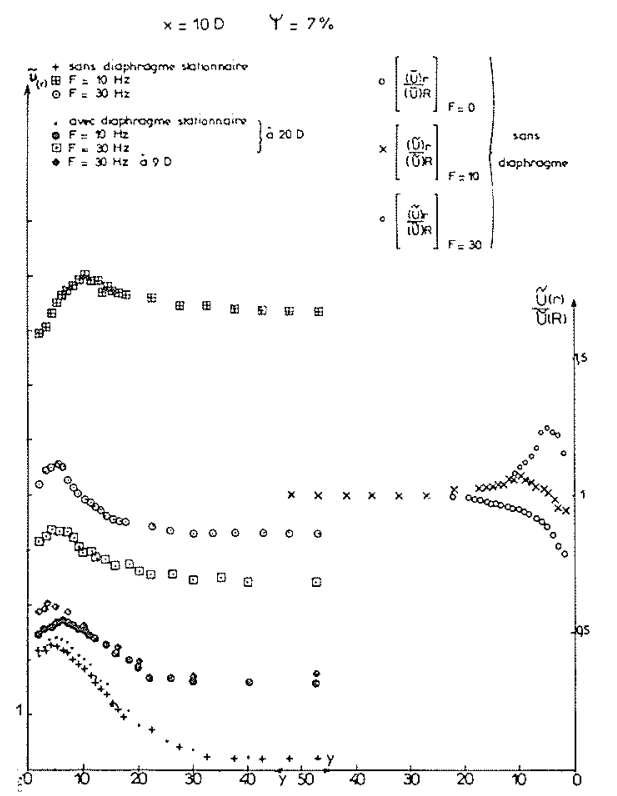

Figure 10

$\left(S_{T}=0,6 \cdot 10^{-2}\right)$ soit beaucoup plus petit que la fréquence caractéristiques des grosses structures turbulentes. Cette modification ne peut être analysée qu'avec un modèle bidimensionnel de l'écoulement turbulent pulsé. Celui-ci a été construit en considérant le gradient de pression transversal nul. Il résoud l'équation de Navier Stokes, l'équation de continuité et le modèle de Bradshaw. Ce dernier fait intervenir une loi empirique et l'équation de transport de l'énergie cinétique de la turbulence $q^{2}$ avec l'hypothèse de proportionnalité entre le frottement et l'énergie cinétique. Le modèle bidimensionnel de l'écoulement turbulent pulsé en canalisation cylindrique est en cours d'analyse et de comparaison avec les grandeurs expérimentales des fluctuations de vitesse longitudinale indiquées sur la figure 10 ; - soit à la réfraction des ondes de pression à la frontière de la couche limite.

La dépendance du coefficient $f$ au taux de modulation de débit constatée lors de l'étude de l'entrée à bord vif semble devoir être mise au compte de la perte de charge singulière d'entrée.

\section{Perte de charge singulière du diaphragme en écoulement turbulent pulsé (fig. 2b)}

La connaissance de la perte de charge régulière dans la canalisation cylindrique en écoulement turbulent pulsé permet d'analyser la contribution de la perte de charge singulière du diaphragme. Cette dernière est dépendante du profil de vitesse longitudinale en écoulement stationnaire. Il est donc nécessaire de placer le diaphragme dans le domaine où ces profils évoluent $(x<60 D)$.

\section{Résultats obtenus}

Sur les figures $11 \mathrm{a}$ et $\mathrm{b}$ est reportée l'évolution des fluctuations de pression en fonction de la fréquence pulsatoire dans les plans d'abscisses respectives $x=D$ et $x=10 D$ lorsque le diaphragme est placé respectivement à $10 D, 20 D, 30 D$ et $50 D$ de l'entrée profilée.

Nous pouvons noter sur ces figures que les modes acoustiques de l'installation sont modifiés. Lorsque le diaphragme est placé dans la $1^{\text {ère }}$ moitié de la longueur de la canalisation, le $1^{\mathrm{eT}}$ mode de résonance disparaît. Lorsqu'il est placé dans la $2^{\text {ème }}$ moitié, c'est le $2^{\text {ème }}$ mode qui est affecté.

Sur la figure 9 sont reportées les fluctuations de pression en fonction de l'abscisse réduite pour les différentes positions du diaphragme. Nous notons qu'au fur et à mesure que le diaphragme s'éloigne de l'entrée l'amortissement des fluctuations de pression diminue.

La surestimation de débit demeure identique à celle indiquée précédemment. Elle est donc indépendante de la forme d'entrée. La modification du coefficient de débit peut être dépendante :

- soit des caractéristiques acoustiques des ondes de pression à longue distance dont les amplitudes sont fortement amorties par la perte de charge singulière. A une telle hypothèse correspondraient une modi-
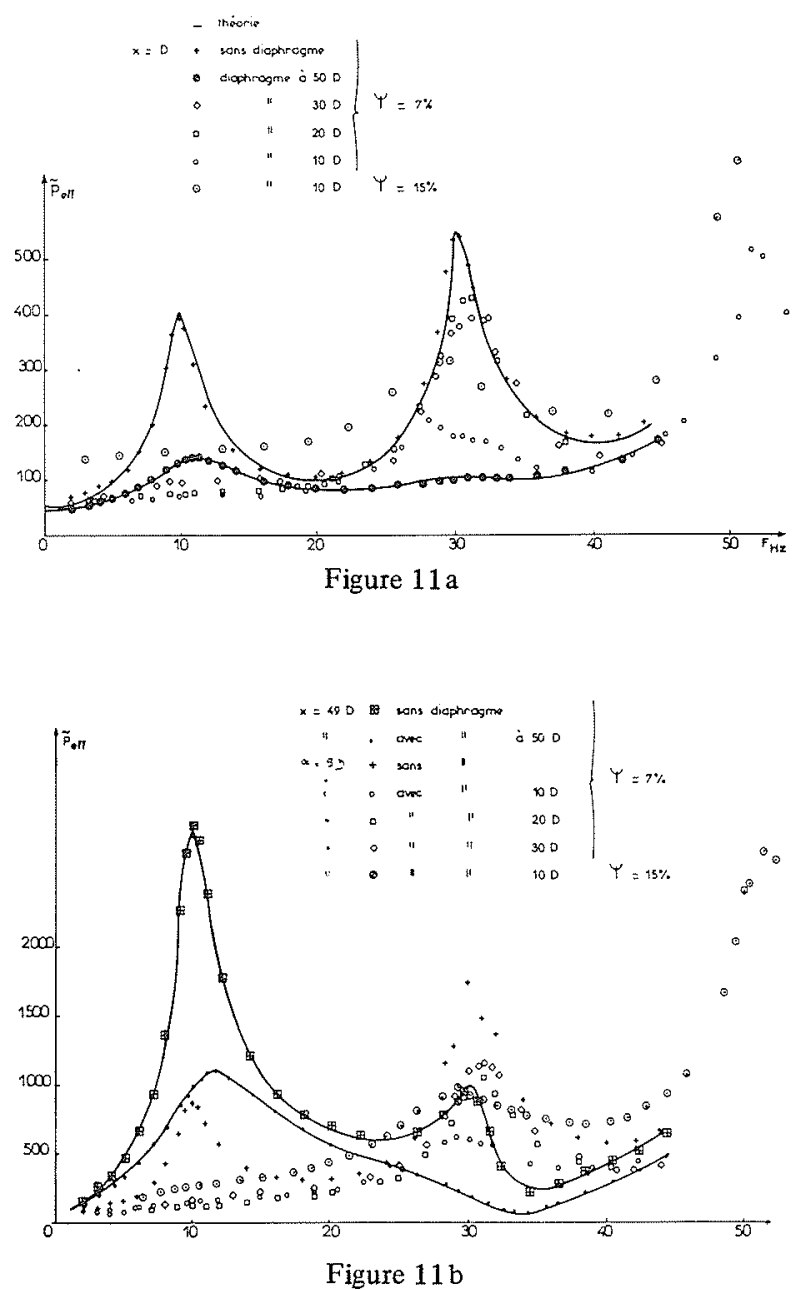
fication de la pression statique moyenne et une altération des profils de vitesse longitudinale en amont du diaphragme. Celles-ci n'ont pas été constatées expérimentalement ;

- soit des caractéristiques acoustiques à courte distance des ondes de pression incidentes et réfléchies à travers le diaphragme. A cette $2^{\text {ème }}$ hypothèse correspondraient une modification de la pression statique moyenne dans le plan de section minimale du jet et une altération du profil de vitesse longitudinale dans ce plan. Celles-ci ont été constatées expérimentalement.

\section{Conclusions}

La surestimation de débit constatée lorsque le taux de modulation de débit est égal à $15 \%$ semble correspondre à la "respiration" du bulbe en aval du diaphragme. Celleci peut être créée par l'influence des caractéristiques acoustiques des ondes de pression en aval du diaphragme. Cette hypothèse, à vérifier, ne semble pas confirmée puisque cette surestimation est indépendante de la position du diaphragme dans l'installation. Cette "respiration" peut être due à la modification de la frontière du jet dépendante de l'arête vive du diaphragme. Cette hypothèse entraînerait une modification des mécanismes turbulents dans le jet où le nombre de Strouhal turbulent est élevé $\left(S_{T}=0,15\right)$.

Cette étude a montré que, pour les zones où le gradient de pression longitudinal n'est pas constant, les valeurs moyennes de l'écoulement turbulent pulsé sont altérées. La perte de charge singulière du diaphragme en est une autre justification puisque ces coefficients aérauliques sont modifiés. Il semble utile d'analyser l'influence de ce gradient correspondant à d'autres pertes de charge singulière (coude, élargissement, jonction).

Les caractéristiques des ondes de pression sont altérées par une perte de charge singulière. Cette modification due à la présence d'un orifice dans une installation peut être utilisée pour éviter les mises en résonance des canalisations.

\section{Notations :}

$A$ : $\quad$ aire de la section droite du conduit
$A_{c}$ : aire de la section droite du col sonique

$C_{D n}$ :Coefficient de débit du diaphragme - norme "AFNOR-X-10110"

$D$ : diamètre de la canalisation

$f: \quad$ coefficient de perte de charge $\left(f=\frac{\epsilon_{c}}{2 D}\right)$

$k$ : coefficient aléatoire associé à la perte de charge d'un diaphragme noyé

$K_{3}=\left(\frac{2}{\gamma+1}\right)^{\gamma+1 / 2(\gamma-1)} \cdot \gamma^{1 / 2} \cdot \frac{\bar{\rho}}{p_{0}^{1 / 2 \gamma}}$

$m$ : coefficient d'obturation du diaphragme $\left(m=(d / D)^{2}\right)$ où $d$ est le diamètre de l'ouverture du diaphragme

$p, P:$ pression, indice $i$ indique la pression génératrice

$\Delta p:$ perte de charge

$t:$ temps

$u: \quad$ vitesse longitudinale

$x$ : abscisse - sens de l'écoulement

$\partial:$ dérivée partielle

$\epsilon: \quad$ coefficient de compressibilité $\left(\epsilon=p_{2} / p_{1}\right)$

$\xi_{c}$ : coefficient de perte de charge régulière linéique

$\gamma: \quad$ rapport des chaleurs massiques $(\gamma=1,4)$

$\rho:$ masse volumique

Indices supérieures

quantité fluctuante périodique

- quantité moyenne temporelle.

\section{Références}

[1] MAINARDI H. - Etude théorique et expérimentale d'une perte de charge singulière en écoulement pulsé. Thèse Doct. Ing. Univ. Orléans, 1975.

[2] MAINARDI H., BARRIOL R., PANDAY P.K. - Pulsating duct flow in the presence of an orifice plate, Int. J. mech. Sci., Vol. 19, pp. 533-546 Pergamon Press, 1977.

[3] NELSON R.M. and PLETCHER R.H. - An explicit scheme for the calculation of confined turbulents with heat transfert. Proc. Heat Transfer and Fluid. Mech. Corvallis University Oregon, June 1974. 


\section{Discussion}

M. le Président. - Je remercie M. MAINARDI de cet exposé très clair et très intéressant.

On rencontre souvent des problèmes de mesure de débit dans des installations qui ne sont pas tout à fait conformes aux recommandations des normes. Les écoulements sont plus turbulents qu'on ne le voudrait aussi.

M. GUITON. - A quoi faites-vous allusion en parlant de "respiration de l'essai" ? Est-ce à la tendance à pulser, ou à la tendance à se déplacer en accordéon?

M. MAINARDI. - La respixation en question est "la respiration du bulbe en aval du diaphragme". Quand nous pulsons avec un taux de modulation de débit de $15 \%$, la section minimale du jet situé à un demi diamètre en aval du diaphragme diminue de $3 \%$ d'une part, et d'autre part la modulation de pression en aval semble indiquer que le point de recollement du jet, situé à dix diamètres de conduit en aval du diaphragme en écoulement stationnaire, est situé à six diamètres. Nous pensons que ce sont les effets acoustiques en aval du diaphragme qui ont pour effet de produire la "respiration" du bulbe.

La prospection des réponses amont des modulations de pression et de vitesse longitudinale, comparée à celles de l'installation démunie du diaphragme, ont montré l'effet d'atténuation provoquée par ce filtre. Le modèle théorique rendant compte de ces modulations prend en compte la majoration du coefficient de frottement équivalent de l'écoulement turbulent pulsé et du coefficient de débit. Lorsque le taux de modulation de débit est de $15 \%$, le coefficient de débit est diminué de $4,5 \%$ quelle que soit la longueur aval. Par conséquent c'est inhérent à la zone du bulbe puisque l'effet de compressibilité à l'aval varie en tant qu'amplitude et que la même constatation a lieu quelle que soit la longueur aval de la canalisation.

L'étude de la réponse du jet à $15 \%$ est en cours. Son analyse nécessiterait de la comparer avec des taux supérieurs tels que $19 \%$ et $30 \%$.

L'intérêt sera ensuite de comparer ces résultats à ceux d'une installation du type industriel puisque notre installation expé- rimentale ne donne que le signal fondamental de la modulation de débit.

M. CHAUVIN. - Je voudrais signaler une possibilité de collaboration:

Nous effectuons dans un tout autre domaine un calcul par résolution des équations de Navier-Stokes pour des écoulements en diaphragmes simples ou multiples. D'autre part, dans le cadre d'études biomécaniques, nous avons des installations expérimentales qui permettent de mesurer des écoulements pulsés de type pratiquement quelconque dans une gamme assez grande d'amplitude et de fréquence.

M. MAINARDI. - Je remercie M. CHAUVIN de son offre qui me serait utile pour analyser certaines conclusions. Notre cas se rapporte à des écoulements gazeux dont le nombre de Reynolds est de l'ordre de $1,5 \cdot 10^{5}$. Un modèle bidimensionnel en cours d'analyse résoud les équations de Stokes avec une loi de fermeture indiquée par BRADSHAW. Les contraintes tangentielles turbulentes sont proportionnelles à l'énergie cinétique turbulente. Cette analyse est effectuée dans le cas de la perte de charge régulière dans le domaine d'établissement. Lorsque le diaphragme est placé dans la canalisation il sera nécessaire de modéliser la "turbulence" du jet. Nous avons constaté expérimentalement que l'épaisseur de couche limite en amont du diaphragme était modifiée, en écoulement stationnaire, sur cinq diamètres en amont du diaphragme. Pour rendre compte de cette modification nous avons pensé utiliser la loi de VAN DRIEST qui permet de rendre compte du frottement rugueux. Ceci nous amènera à considérer une rugosité théorique qui rend compte de la modification des profils de vitesse longitudinale dans ce domaine, et de la majoration du gradient de pression constatée sur deux diamètres en amont. Nous tentons cette astuce parce qu'il faut pouvoir traduire l'effet d'aplatissement des profils de vitesse en amont du diaphragme. Je ne sais comment ce sera admis par les grands physiciens mais il nous faut connaître cette discontinuité dans le domaine deux diamètres en amont et six diamètres en aval du diaphragme, domaine qui caractérise la perte de charge singulière et le jet confiné en théorie bidimensionnelle. 